\title{
High glucose inhibits the osteogenic differentiation of periodontal ligament stem cells in periodontitis by activating endoplasmic reticulum stress
}

\author{
Jun Tan ${ }^{1 \#}$, Yang Zhou ${ }^{2 \#}$, Jing Luo ${ }^{1}$, Xiaoxue Wu ${ }^{3}$, Haibo Liu', Weina Wang ${ }^{1}$, Zebin Li ${ }^{1}$, Mengyi Zhong ${ }^{1}$, \\ Lijing $\mathrm{Wu}^{1}$, Xiao $\mathrm{Li}^{1}$ \\ ${ }^{1}$ Department of Stomatology, General Hospital of Southern Theater Command of the Chinese People's Liberation Army (Guangzhou Liuhuaqiao \\ Hospital), Guangzhou, China; ${ }^{2}$ Department of Anesthesiology, General Hospital of Southern Theater Command of the Chinese People's Liberation \\ Army (Guangzhou Liuhuaqiao Hospital), Guangzhou, China; ${ }^{3}$ Department of Orthodontics, Key Laboratory of Oral Medicine, Guangzhou Institute \\ of Oral Disease, Stomatology Hospital of Guangzhou Medical University, Guangzhou, China \\ Contributions: (I) Conception and design: J Tan, Y Zhou, X Li; (II) Administrative support: X Li; (III) Provision of study materials or patients: J Luo, \\ X Wu, H Liu, W Wang, Z Li, M Zhong, L Wu; (IV) Collection and assembly of data: J Tan, Y Zhou, J Luo; (V) Data analysis and interpretation: J \\ Tan, Y Zhou, J Luo; (VI) Manuscript writing: All authors; (VII) Final approval of manuscript: All authors. \\ \#These authors contributed equally to this work as co-first authors. \\ Correspondence to: Xiao Li. Department of Stomatology, General Hospital of Southern Theater Command of the Chinese People's Liberation Army \\ (Guangzhou Liuhuaqiao Hospital), No. 111, Liuhua Road, Guangzhou 510016, Guangdong, China. Email: drlixiao@163.com.
}

Background: Periodontitis is a highly prevalent dental disease which is associated with diabetes and is challenging to cure in diabetic patients. However, the mechanism of comorbid diabetes and periodontitis is still unclear. This study aimed to uncover the role of endoplasmic reticulum (ER) stress in high glucoseassociated periodontitis.

Methods: Periodontal tissues were obtained from diabetic patients with periodontitis, periodontitis patients without systemic disease, and healthy teeth. The expressions of ER stress-related factors GRP78, ATF6, PERK and XBP1 were detected by quantitative real-time polymerase chain reaction (qRT-PCR) and immumohistochemical staining. Periodontal ligament stem cells (PDLSCs) from three states of periodontal tissues were isolated and cultured as diabetic PDLSCs (dPDLSCs), inflamed PDLSCs (iPDLSCs) and healthy PDLSCs (hPDLSCs), and the cell stemness was assayed. Different concentrations (8, 11, and $25 \mathrm{mmol} / \mathrm{L}$ ) of D-glucose were used on hPDLSCs to simulate high glucose microenvironment. The changes of osteogenic ability of PDLSCs were observed, and the expressions of ER stress-related factors in different time point (6, 12, 24, and 72 h) were detected. Finally, GRP78 shRNA lentivirus was used to block ER stress on PDLSCs in the $25 \mathrm{mmol} / \mathrm{L}$ D-glucose microenvironment, and the osteogenic ability of PDLSCs was observed.

Results: The results showed that the expressions of GRP78, ATF6, PERK, and XBP1 were highest in the diabetic periodontitis group and lowest in the healthy periodontal tissue group $(\mathrm{P}<0.05)$. The clone formation, osteogenic and lipogenic differentiation abilities were lowest in dPDLSCs and highest in hPDLSCs. With the increase of glucose concentration, the osteogensis ability of PDLSCs decreased. After 6 hours of stimulation with D-glucose $25 \mathrm{mmol} / \mathrm{L}$, the ER stress pathways in PDLSCs were effectively activated, and the peak value was reached at 12 hours. The decrease in the osteogensis ability of PDLSCs in a high glucose microenvironment reversed when ER stress was blocked.

Conclusions: The osteogenic differentiation ability of PDLSCs cells is inhibited in a high glucose microenvironment, and this effect is realized by ER stress activation. Blocking ER stress can partially restore the reduced osteogenic ability of PDLSCs. These results suggest that high glucose inhibits the osteogenic differentiation ability of PDLSCs by activating ER stress, which ultimately exacerbates periodontitis.

Keywords: Periodontitis; endoplasmic reticulum stress; periodontal ligament stem cells (PDLSCs); osteogenesis; diabetes 
Submitted Nov 12, 2021. Accepted for publication Feb 21, 2022.

doi: $10.21037 / \mathrm{atm}-22-6$

View this article at: https://dx.doi.org/10.21037/atm-22-6

\section{Introduction}

Periodontitis can cause pathological loss of the periodontal ligament and alveolar bone (1). Without timely treatment, the alveolar bone loss will progress, eventually leading to tooth looseness, which can seriously affect people's health. There are many risk factors for periodontitis, such as smoking, drinking, immune deficiency, and osteoporosis (2-4). Diabetes is also considered to be an important aggravating factor for periodontal disease, with periodontal disease being listed as the sixth complication of diabetes (5). A two-way interaction is believed to exist between type 2 diabetes and periodontitis. Type 2 diabetes can prompt a high inflammatory reaction, disrupt the bone repair process, and produce advanced glycation end products, leading to the production and further deterioration of periodontitis (6-8). Meta-analyses of adjusted estimates showed that diabetes increased the risk of incidence or progression of periodontitis by $86 \%$ [RR 1.86 (95\% CI: 1.3-2.8)] (9). In a study of 350 diabetic children (6-18 years old) vs. 350 non-diabetic controls, the proportion of periodontal sites with evidence of periodontitis was greater in the children with diabetes $(>20 \%$ vs. $8 \%$ of sites, respectively) (10). Meanwhile, periodontitis may make it more difficult to control the blood glucose of diabetic patients and increase the risk of diabetes complications (11). A study shows that effective treatment of periodontitis can improve the glucose metabolism of patients with type 2 diabetes, which is beneficial to reducing complications related to hyperglycemia (12). Therefore, control of blood glucose concentration and basic periodontal treatment are beneficial in the treatment of type 2 diabetic patients with periodontitis. Various biologically plausible molecular-level mechanisms have been established about the associations between high glucose and periodontitis (13), while fewer researches focused on organelle level.

Periodontal ligament stem cells (PDLSCs) are heterogeneous mesenchymal stem cells (MSCs) located in the periodontal ligament, which are the key cells maintaining the structure and function of the periodontal ligament. They could be identified by colony forming ability and multipotential differentiation ability, such as osteogenic and lipogenic differentiation abilities. Previous research found that PDLSCs could differentiate into periodontal membrane cells and cementum cells in vitro and in vivo to form periodontal and cementum structures $(14,15)$. However, in diabetic patients with periodontitis, the ability of PDLSCs to repair and rebuild periodontal membrane tissue is greatly reduced, and the therapeutic effect is weakened (16). Endoplasmic reticulum (ER) stress, which widely occurs in eukaryotic cells, is a protective stress response induced by external stimulus such as ischemia, hypoxia, glycopenia and infection so as to cause the stacking of unfolded proteins or misfolded proteins in ER. ER stress mainly involves three unfolded protein response (UPR) signaling pathways, including activating transcription factor 6 (ATF6) pathway, double-stranded RNA-activated protein kinase (PKR) like endoplasmic reticulum kinase (PERK) pathway, and inositol requiring enzyme 1 (IRE1) pathway. The ER stress marker is ER luminal binding protein chaperone-binding immunoglobulin protein/ glucose regulated protein 78 (BiP/GRP78), and the UPR pathway markers are ATF6, PERK, and X boxbinding protein 1 (XBP1) respectively. While moderate ER stress can effectively protect the body, excessive ER stress damages it (17). Diabetes can disrupt ER function, which causes an imbalance of ER homeostasis, resulting in ER stress (18). A sdudy showed that apoptosis induced by ER stress was involved in the effect of periodontitis on vascular calcification in a rat model (19), but researches on human PDLSCs were not involved. In our previous study, ER stress was also found to participate in reducing the osteogenic differentiation ability of PDLSCs in the inflamed microenvironment induced by tumor necrosis factor- $\alpha$ (20), which indicated ER stress could promote the damage of periodontal tissue in periodontitis. The present study focused on the ER stress response at the organelle level. ER stress activation was analyzed in periodontal tissues in different states, and the osteogenic differentiation ability was compared among PDLSCs in different states. Then, the influence of high glucose on the osteogenic differentiation ability of PDLSCs and the changes in ER stress-related factors were detected, with the aim of providing a new target for the treatment of diabetic patients with periodontitis. 
Table 1 Grouping of research individuals

\begin{tabular}{|c|c|c|c|c|c|c|c|c|c|}
\hline Groups & \multicolumn{3}{|c|}{ qRT-PCR } & \multicolumn{3}{|c|}{ Immunohistochemical test } & \multicolumn{3}{|c|}{ Cell culture } \\
\hline Sample size & 10 & 10 & 8 & 8 & 8 & 8 & 6 & 6 & 8 \\
\hline Gender (M/F) & $5 / 5$ & $7 / 3$ & $4 / 4$ & $4 / 4$ & $7 / 1$ & $5 / 3$ & $4 / 2$ & $3 / 3$ & $5 / 3$ \\
\hline Age $(Y$, mean $\pm S D)$ & $50.5 \pm 7.3$ & $53.7 \pm 6.0$ & $27.9 \pm 3.4$ & $49.6 \pm 6.3$ & $53.6 \pm 5.6$ & $27.5 \pm 3.6$ & $50.2 \pm 4.4$ & $52.8 \pm 5.0$ & $26.5 \pm 3.2$ \\
\hline
\end{tabular}

qRT-PCR, quantitative real-time polymerase chain reaction; D, diabetic group; I, inflamed group; H, healthy group; M, male; F, female; Y, years; SD, standard deviation.

We present the following article in accordance with the MDAR reporting checklist (available at https://atm. amegroups.com/article/view/10.21037/atm-22-6/rc).

\section{Methods}

\section{Materials}

Periodontal tissues on the root surface were obtained from the affected teeth of diabetic patients with periodontitis (the diabetic periodontitis group, diabetic group for short) and periodontitis patients without systemic disease (the inflamed periodontal tissue group, inflamed group for short). In addition, periodontal ligaments (the healthy periodontal tissue group, healthy group for short) were obtained from healthy premolars or buried third molars extracted during orthodontic procedures. Affected teeth and healthy teeth were also collected. The teeth were collected from patients in Department of Stomatology in General Hospital of Southern Theater Command of the Chinese People's Liberation Army, and Department of Orthodontics in Stomatology Hospital of Guangzhou Medical University, and all the tooth extractions were performed in Department of Stomatology in General Hospital of Southern Theater Command. Teeth in each group were divided randomly into three groups for gene expression analysis, immunohistochemical test, and cell culture (for grouping, see Table 1). None of the study participants had systemic diseases or a long-term medication history except diabetes, and none of them had received periodontal treatment within the previous 3 months. All procedures performed in this study involving human participants were in accordance with the Declaration of Helsinki (as revised in 2013). The experimental protocol was approved by the Medical Ethics Committee of the former Guangzhou General Hospital of Guangzhou Military Command (General Hospital of
Southern Theater Command of the Chinese People's Liberation Army) (No. 2017-3-6). All study participants gave informed consent to undergo sampling. All tooth extraction regimens were in accordance with the oral treatment protocol.

PDLSCs were derived from the periodontal tissues of three aforementioned groups. Periodontal tissues were digested using explants with enzymatic digestion method to obtain the cell suspension as previously described (20). Briefly, PDL or granulation tissues were gently scraped from the middle third of the root at the time of extraction of teeth. The tissues were cut into $1 \mathrm{~mm}^{3}$ cubes, washed by phosphate buffer saline (PBS) supplemented with $100 \mathrm{U} / \mathrm{mL}$ penicillin and $100 \mathrm{mg} / \mathrm{mL}$ streptomycin for three times, digested by $3 \mathrm{mg} / \mathrm{mL}$ collagenase type I for $40 \mathrm{~min}$ at $37^{\circ} \mathrm{C}$ and placed into six-well culture dishes. The tissues were cultured in $\alpha$-minimum essential medium ( $\alpha$-MEM) (Gibco, Grand Island, USA, C12571500BT) supplemented with $10 \%$ fetal bovine serum (FBS) (Gibco, 10099133), $100 \mathrm{U} / \mathrm{mL}$ penicillin $\mathrm{G}$ and $100 \mathrm{mg} / \mathrm{mL}$ streptomycin, at $37{ }^{\circ} \mathrm{C}$ in $5 \% \mathrm{CO}_{2}$. To gain purified homogeneous populations of PDLSCs, limiting dilution technique was used to obtain single cell derived colony cultures from primary cells. The culture medium was refreshed every 3 days, and cells were subcultured at approximately 80-90\% confluence with trypsin. The subcultured cells at passages 3-5 (P3-P5) were used in following experiments. D-glucose (Macklin Inc., Shanghai, China, D810588) was added into culture medium to simulate high glucose microenvironment. GRP78 short hairpin RNA (shRNA) lentivirus vector (Ribo Bio Co, Guangzhou, China) was constructed and sequenced, and the sequencing was confirmed to be correct. The sequence used was as follows: Forward: TTAGAUCAUAGUUACGCGAGG Reverse: TTCCUCGCGUAACUAUGAUCU. 


\section{Quantitative real-time polymerase chain reaction (qRT-PCR)}

The obtained periodontal tissues were homogenized to flocculent in $1 \mathrm{ml}$ RNAiso Plus on ice using an electric homogenizer. PDLSCs cultured in 6-well plates were washed three times with pre-cooling phosphate-buffered saline (PBS) and added by $300 \mu \mathrm{L}$ RNAiso Plus per well. Each periodontal tissue or sample of cells was lysed and transfered to an enzyme-free EP tube with chloroform in $1 / 5$ volume of RNAiso Plus. The EP tube was shake violently for $15 \mathrm{~s}$, and stand at room temperature (RT) for 5 min until the liquid is preliminarily layered. Centrifugation was performed at $12,000 \mathrm{rpm}$ at $4{ }^{\circ} \mathrm{C}$ for 15 minutes. The aqueous phase containing RNA was taken and mixed with isopyknic isopropyl alcohol and placed on ice for 15 minutes. The centrifuged at $12,000 \mathrm{rpm}$ at $4{ }^{\circ} \mathrm{C}$ for a further 15 minutes, after which the supernatant was discarded. Then, $1 \mathrm{~mL} 75 \%$ ethanol (prepared with double-distilled $\mathrm{H}_{2} \mathrm{O}$ ) was added and centrifuged at $4{ }^{\circ} \mathrm{C}$ at $10,000 \mathrm{rpm}$ for 5 minutes, and the supernatant was once again discarded. The obtained RNA was dried and quantified, and $1 \mu \mathrm{g}$ RNA was reverse transcribed into complementary DNA (cDNA) using the miScript Reverse Transcription Kit (Qiagen, Beijing, China, KR118) in strict accordance with the instructions. The cDNA was diluted with double-distilled $\mathrm{H}_{2} \mathrm{O}$. A $10-\mu \mathrm{L}$ system consisting of $2.5 \mu \mathrm{L}$ cDNA, $5 \mu \mathrm{L}$ fluorescent dye SYBER Green (Qiagen, Beijing, China), $0.5 \mu \mathrm{L}$ upstream and downstream primers, and $1.5 \mu \mathrm{L}$ diethylpyrocarbonate (DEPC)-treated water was used for amplification on a MyCycler ${ }^{\mathrm{TM}}$ Real-Time PCR Detection System (BioRad Laboratories, Inc, Richmond, CA, USA). Finally, the obtained data were imported into statistical software for analysis.

The primer (Generay Biotech, Shanghai, China) sequences used were as follows:

GRP78: Forward: CCTTCGATGTGTCTCTTCTCAC Reverse: ACGCTGGTCAAAGTCTTCTC;

ATF6: Forward: TACCTGGGAGTCTGGAGTTT Reverse: CCCAGGATAGCCAAATCTAAGG; PERK: Forward: GGAGGACAAGTAGCACAAACT

Reverse: GTAAACCCACCCTAGAACCATC; XBP1: Forward: GAGACAGAGAGCCAAGCTAATG Reverse: CAGGTTCTTCCTTCACTGAGAC; RUNX2: Forward: GCTTCATTCGCCTCACAAAC Reverse: GTAGTGACCTGCGGAGATTAAC;

OCN: Forward: TCACACTCCTCGCCCTATT Reverse: CCTCCTGCTTGGACACAAA;

\section{GAPDH: Forward: CAAGAGCACAAGAGGAAGAGAG} Reverse: CTACATGGCAACTGTGAGGAG.

\section{Immunobistochemical test}

The teeth with complete periodontal ligament were fixed with $4 \%$ paraformaldehyde, embedded in paraffin, decalcified by $10 \%$ EDTA-2Na, and sectioned at a thickness of $5 \mu \mathrm{m}$. The tissue sections were placed on a slide, transferred to a thermostat, and baked at $60{ }^{\circ} \mathrm{C}$ for 1 hour until the wax on the surface of the tissue sections had melted. The slices were treated with xylene for 15 minutes and dewaxed. After dewaxing, the tissue sections were soaked in $100 \%$ ethanol for 5 minutes, and then in $95 \%, 90 \%, 80 \%$, and $70 \%$ ethanol for 2 minutes each time, before being washed with PBS three times. The tissue sections were subsequently placed in a pressure cooker, to which citrate buffer was added, and boiled for 5 minutes for antigen repair. The tissue sections were first washed with distilled water and PBS twice, and then blocked with $5 \%$ bovine serum albumin for 10 minutes. Specific primary antibody was added to the tissue slices ahead of incubation overnight at $4{ }^{\circ} \mathrm{C}$. After three washes with PBS, the secondary antibody was added, and the tissue sections were incubated at $37^{\circ} \mathrm{C}$ for 2 hours and then washed three times with PBS. The sections were subjected to Diaminobenzidine (DAB) color development for 5 minutes, washed with distilled water to remove float color, and redyed with hematoxylin for 10 seconds. Gradient ethanol was used to dehydrate the slices for 2 minutes at each concentration and the slices were hyalinized by xylene for 5 minutes. Finally, the plates were sealed with resin and observed under a microscope and photographed.

The antibodies used are as follows: rabbit anti-GRP78 (1:1,000 dilution, Cell Signaling Technology, Beverly, USA, 3177S), rabbit anti-ATF6 (1:1,000 dilution, Abcam, Cambridge, UK, ab122897), rabbit anti-PERK (1:1,000 dilution, CST, 3192S), rabbit anti-XBP1 (1:1,000 dilution, Abcam, ab109221).

\section{Cell stemness assay}

Cell cloning was performed as follows. The thirdgeneration PDLSCs from the three sources were collected, digested with $0.25 \%$ trypsin for 5 minutes, centrifuged at $1,000 \mathrm{rpm}$ for 10 minutes, and washed twice with PBS. After being resuspended, the cells were inoculated on a 6-well plate with the cell density adjusted to 200 cells/well. 
Following 14 days of culture under suitable conditions, the cells were fixed with $4 \%$ paraformaldehyde, and then dyed with $0.1 \%$ crystal violet solution for 10 minutes. Finally, the staining solution was removed, photos were taken, and the clone formation rates of PDLSCs were calculated.

Osteogenic induction was performed as follows. PDLSCs were inoculated in 6-well plates at a density of $1 \times 10^{5}$ cells/ well. To induce osteogenesis, $10 \mathrm{mmol} / \mathrm{L} \beta$-glycerophosphate, $1 \mu \mathrm{mol} / \mathrm{L}$ dexamethasone, and $50 \mu \mathrm{mol} / \mathrm{L}$ vitamin $\mathrm{C}$ were added into the culture medium. After 4 week-induction, the cells were rinsed twice with PBS, fixed with $95 \%$ ethanol for 10 minutes, hydrated with distilled water for $2-3 \mathrm{~min}$, and stained with $0.1 \%$ alizarin red dye at RT for 3 minutes. The dye was washed off, and the cells were washed twice with PBS. Finally, the cells were observed under a microscope and photographed for analysis.

Adipogenic induction was performed as follows. PDLSCs were inoculated into 6-well plates at a density of $1 \times 10^{5}$ cells/well. To induce adipogenesis, the medium was supplemented with $0.5 \mathrm{mmol} / \mathrm{L}$ isobutylmethylxanthine, $200 \mu \mathrm{mol} / \mathrm{L}$ indomethacin, $1 \mu \mathrm{mol} / \mathrm{L}$ dexamethasone, and $10 \mu \mathrm{mol} / \mathrm{L}$ insulin. Four weeks after adipogenic induction, the cells were rinsed twice with PBS, fixed with $60 \%$ isopropyl alcohol for 1 minute, hydrated with distilled water for 2 3 min, stained with oil red $\mathrm{O}$ solution for 3 minutes. Finally, after being washed twice with PBS, the cells were observed under a microscope (Olympus, Tokyo, Japan) and photographed for analysis.

\section{Alkaline phosphatase (ALP) activity assay}

PDLSCs were inoculated in 12-well plates at a density of $1 \times 10^{5}$ cells/well, and induced by osteogenic culture for one week. The cells were fixed rinsed twice with PBS, fixed with $4 \%$ paraformaldehyde at RT for 30 minutes, and then rinsed twice with PBS again. ALP staining was performed by BCIP/NBT ALP chromogenic Kit (Beyotime Biotechnology, Shanghai, China, C3206) according to the manufacturer's instructions. ALP staining densities were measured by an automated image-analysis system.

\section{Western blot}

After 14 days of osteogenic induction or high glucose stimulation for a certain length of time, cells were taken from each group, digested with $0.25 \%$ trypsin for 5 minutes, centrifuged at 1,000 rpm for 10 minutes, and then washed twice with PBS. Following resuspension, the cells were centrifuged at $13,000 \mathrm{rpm}$ at $4{ }^{\circ} \mathrm{C}$ for 30 minutes, and the supernatant was collected. Standard protein solution was used to prepare a series of protein solutions with concentration gradients. Then, $10 \mu \mathrm{L}$ of Coomassie Bright Blue dye was added to $100 \mu \mathrm{L}$ of solution at each concentration. Optical density (OD) values were detected with a spectrophotometer, and standard curves were drawn based on the statistical data. To $100 \mu \mathrm{L}$ supernatant, $20 \mu \mathrm{L}$ loading buffer was added boiled at $100{ }^{\circ} \mathrm{C}$ for 5 minutes to denaturate the protein. After being placed on ice for 3 minutes, the samples were centrifuged at $13,000 \mathrm{rpm}$ at $4^{\circ} \mathrm{C}$ for 15 minutes.

Electrophoresis was performed with sodium dodecyl sulphate-polyacrylamide gel electrophoresis (SDSPAGE), running the concentrated gel under $100 \mathrm{~V}$ and the separating gel under $120 \mathrm{~V}$. A polyvinylidene difluoride (PVDF) membrane of an appropriate size was first soaked in methanol for 2 minutes, and then pretreated with transfer solution. After electrophoresis had been completed, the gel was removed and assembled on the PVDF membrane. The membrane was transferred at $0{ }^{\circ} \mathrm{C}$ and $70 \mathrm{~V}$ for 2 hours, covered with $5 \%$ skim milk powder on a shaker for 1 hour, and then incubated with primary antibody at $4{ }^{\circ} \mathrm{C}$ for 24 hours. The protein expression levels of runt-related transcription factor 2 (Runx2) and Osteocalcin (OCN) were detected with specific primary antibodies. The membrane was washed five times with PBS with Tween-20 (PBST), and the secondary antibody was added for incubation for 4 hours. The membrane was again washed with PBST five times and then with pure water five times. The chemiluminescence system was used for 3 minutes, and the images were recorded.

The antibodies used were as follows: rabbit antiRunx2 (1:1,000 dilution, Invitrogen, Thermo Fisher Scientific, Waltham, USA, 702489), rabbit anti-OCN (1:1,000 dilution, Invitrogen, PA5-11849), and mouse anti- $\beta$-actin (1:5,000, Biodragon Immunotech, Beijing, China, B1029). Other antibodies were the same as those in immunohistochemical test.

\section{Statistical analysis}

All experiments were performed three times independently to test for reproducibility, and the data are shown as mean \pm standard deviation (SD). All data in this study were statistically analyzed using GraphPad Prism 8 (GraphPad Software, San Diego, USA). Differences between two groups were evaluated by unpaired two-tailed Student's $t$-test, and comparisons of more than two groups were 


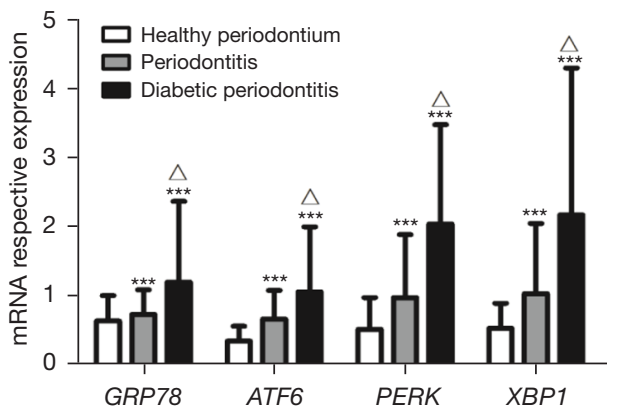

Figure 1 Messenger RNA expression levels of ER stress pathwayrelated factors in periodontal tissues from individuals with healthy teeth, periodontitis patients without systemic diseases, and diabetic patients with periodontitis. ${ }^{* *}, \mathrm{P}<0.001$ compared with healthy group. $\Delta, \mathrm{P}<0.05$ compared with inflamed group. RNA, ribonucleic acid; ER, endoplasmic reticulum.

conducted by one-way analysis of variance (ANOVA) followed by Dunnett's test. $\mathrm{P}<0.05$ was taken to indicate statistical significance.

\section{Results}

\section{The intensity of ER stress in periodontal tissue is increased in diabetic periodontitis}

Messenger RNA (mRNA) was extracted from the periodontal tissues of diabetic patients with periodontitis, periodontitis patients without systemic diseases, and individuals with healthy teeth. Paraffin tissue sections were subjected to immunohistochemical staining. The ER stress-related factors GRP78, ATF6, PERK, and XBP1 were detected. Before immunohistochemical staining, hematoxylin and eosin (HE) staining was performed to confirm that the section area had complete periodontal tissue on the root surface.

Results of qRT-PCR showed that the mRNA expression levels of GRP78, PERK, ATF6, and XBP1 were highest in periodontal tissues of the diabetic group, followed by the inflamed group, and they were lowest in the healthy group. The differences between the three groups were statistically significant $(\mathrm{P}<0.05)$ (Figure 1).

After HE staining of the decalcified sections, the samples were observed under a microscope to identify the parts with intact periodontal tissue. In the healthy group, HE staining showed that the periodontal tissue structure was complete, with collagen fibers that were arranged in order, and a large number of spindle and star-shaped fibrocells arranged along the fiber bundles (Figure 2A). In the inflamed group, HE staining revealed irregularly shaped periodontal ligament with swelling and looseness, a large amount of granulation tissue hyperplasia, and relatively disordered fibroblast arrangement (Figure 2B). In the diabetic group, HE staining showed disordered cell arrangement in the periodontal ligament, with necrotic tissue and infiltration of inflammatory cells (Figure 2C).

Immunohistochemical staining showed that in healthy periodontal tissues, GRP78 was slightly expressed in the perivascular region (Figure $3 A$ ), while ATF6 expression was almost undetectable (Figure $3 B$ ), and PERK and XBP1 were slightly expressed close to the root surface and in some vascular pericytes (Figure 3C,3D). In the inflamed group, GRP78 was widely expressed in perivascular and nonvascular areas (Figure 3E), ATF6 and PERK were expressed in perivascular tissues (Figure $3 F, 3 G$ ), and XBP1 was expressed in periodontal tissues (Figure $3 H$ ). In the diabetic group, ER stress-related factors were widely expressed (Figure 3I,37,3K,3L), and their expression was significantly higher than that in the healthy and inflamed groups (Figure $3 M)$. The expression differences of ER stress-related factors between the three groups were statistically significant (Figure $3 M, \mathrm{P}<0.01$ ).

In summary, the inflammatory response in periodontal tissue was more obvious and the activation intensity of ER stress was significantly higher in the diabetic group than in the inflamed and healthy groups.

\section{The clone formation and multidirectional differentiation ability of PDLSCs is weakened in diabetic periodontitis}

PDLSCs were obtained from periodontal tissue samples of diabetic patients with periodontitis, periodontitis patients without systemic diseases, and individuals with healthy premolars or buried third molars through a process involving enzymatic tissue digestion, primary culture, and cell purification.

The success rate of primary culture of healthy PDLSCs (hPDLSCs) was about $60 \%$. At 3 to 5 days after tissue block inoculation, cells could be seen under a microscope to be migrating out of the tissue block and growing adherent to the wall. The cells were fibroblast-like, long spindle type, with round and centered nuclei (Figure $4 A$ ). After about 2 weeks, the cells reached $80 \%$ confluence. The cell morphology showed no significant change after limited dilution in passage, and the proliferation rate gradually accelerated. The cells reached $80 \%$ confluence again after 

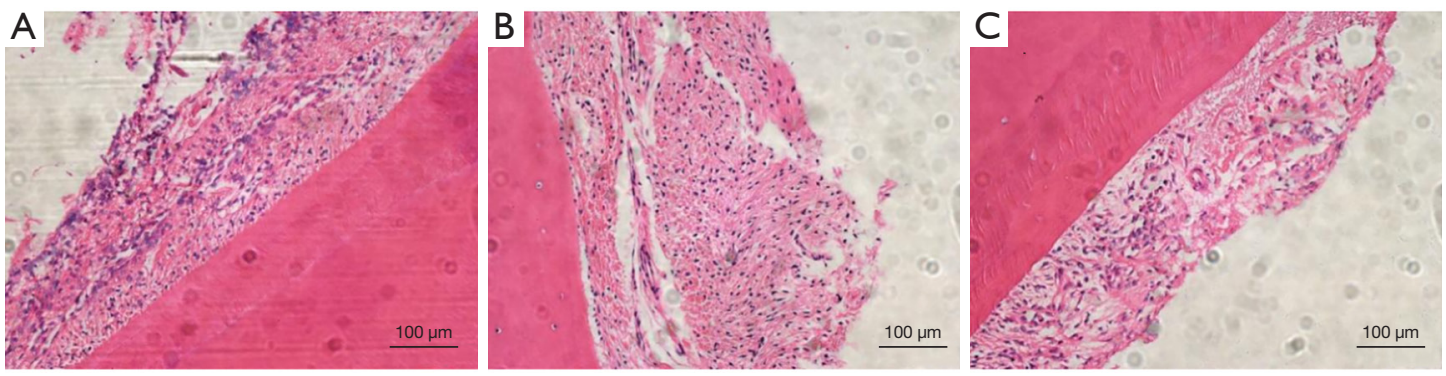

Figure $2 \mathrm{HE}$ staining of periodontal tissues from different sources. (A) HE staining of healthy periodontal tissue. (B) HE staining of periodontal tissue of periodontitis patients without systemic diseases. (C) HE staining of periodontal tissue of diabetic patients with periodontitis. Bar $=100 \mu \mathrm{m}$. HE, hematoxylin and eosin.

about 2 weeks. The proliferation rate of purified secondgeneration PDLSCs (Figure 4B) was significantly faster, and the passage time was 5 to 7 days. The success rate of primary culture of inflamed PDLSCs (iPDLSCs) derived from the periodontal tissue of patients with periodontitis was about $50 \%$. The growth rate of these primary cells was slower than that of hPDLSCs. There was a small number of unextended cells, and the morphology of the extended cells was the same as that of hPDLSCs (Figure 4C). The morphology and proliferation rate of second-generation iPDLSCs after limited dilution and purification were similar to those of hPDLSCs (Figure 4D). The primary culture success rate of diabetic PDLSCs (dPDLSCs) derived from the periodontal tissue of diabetic patients with periodontitis was significantly reduced at approximately $20 \%$. The cell growth rate was significantly lower than that of iPDLSCs. The number of unextended cells was increased, and the morphology of the extended cells was the same as that of hPDLSCs (Figure 4E). After limited dilution and purification, the proliferation rate of second-generation dPDLSCs decreased significantly compared with those of hPDLSCs and iPDLSCs (Figure 4F).

Single-cell suspensions (200 cells) of the third-generation PDLSCs were seeded in 6-well plates and cultured for 14 days. Then, the cells were fixed and stained with crystal violet, which revealed that all three generations of hPDLSCs, iPDLSCs, and dPDLSCs showed clonal proliferation (Figure 5A-5C). The clone formation rates were calculated as $31.5 \% \pm 1.3 \%, 23.8 \% \pm 2.0 \%$, and $16.2 \% \pm 0.8 \%$, respectively, and the differences between the groups were statistically significant $(\mathrm{P}<0.05)$.

About 3 weeks after osteogenic induction, the cells presented as densely formed round nodules under the microscope. After 4 weeks of induction, a large number of pinpoint-sized white nodules could be seen scattered on the bottom of the culture bottle. According to alizarin red staining, a large number of alizarin red mineralized nodules were found in the hPDLSC group (Figure 5D); the alizarin red mineralized nodules in the iPDLSC group were smaller with a reduced area (Figure 5E), and those in the dPDLSC group were significantly reduced with a significantly smaller area (Figure 5F). After approximately 3 weeks of adipogenic induction, transparent vacuole-like structures with beaded arrangement could be observed when the cells were examined under a microscope. After 4 weeks of induction, the number of vacuolar structures were significantly increased. After oil red $\mathrm{O}$ staining, a large number of red lipid droplets, which are indicative of positive staining, were observed in the hPDLSC group (Figure 5G). In comparison, the red lipid droplets in the iPDLSC group were samller and widely formed (Figure 5H), while in the dPDLSC group, the red lipid droplets were significantly fewer and only present in some cells (Figure 5I). The positive rate of both alizarin red staining (Figure 57, $\mathrm{P}<0.001$ ) and oil red $\mathrm{O}$ staining (Figure $5 K, \mathrm{P}<0.001$ ) showed statistically significant differences among the three groups.

In the next part of the experiment, dPDLSCs were successfully isolated and cultured in vitro from residual inflamed periodontal tissue of diabetic patients on the root surface. These cells displayed clone formation ability, and multidirectional differentiation ability of MSCs. However, the cloning, osteogenesis, and lipid formation abilities of dPDLSCs were significantly weaker than those of iPDLSCs extracted from periodontitis patients without systemic diseases, and those of PDLSCs derived from healthy periodontium. 

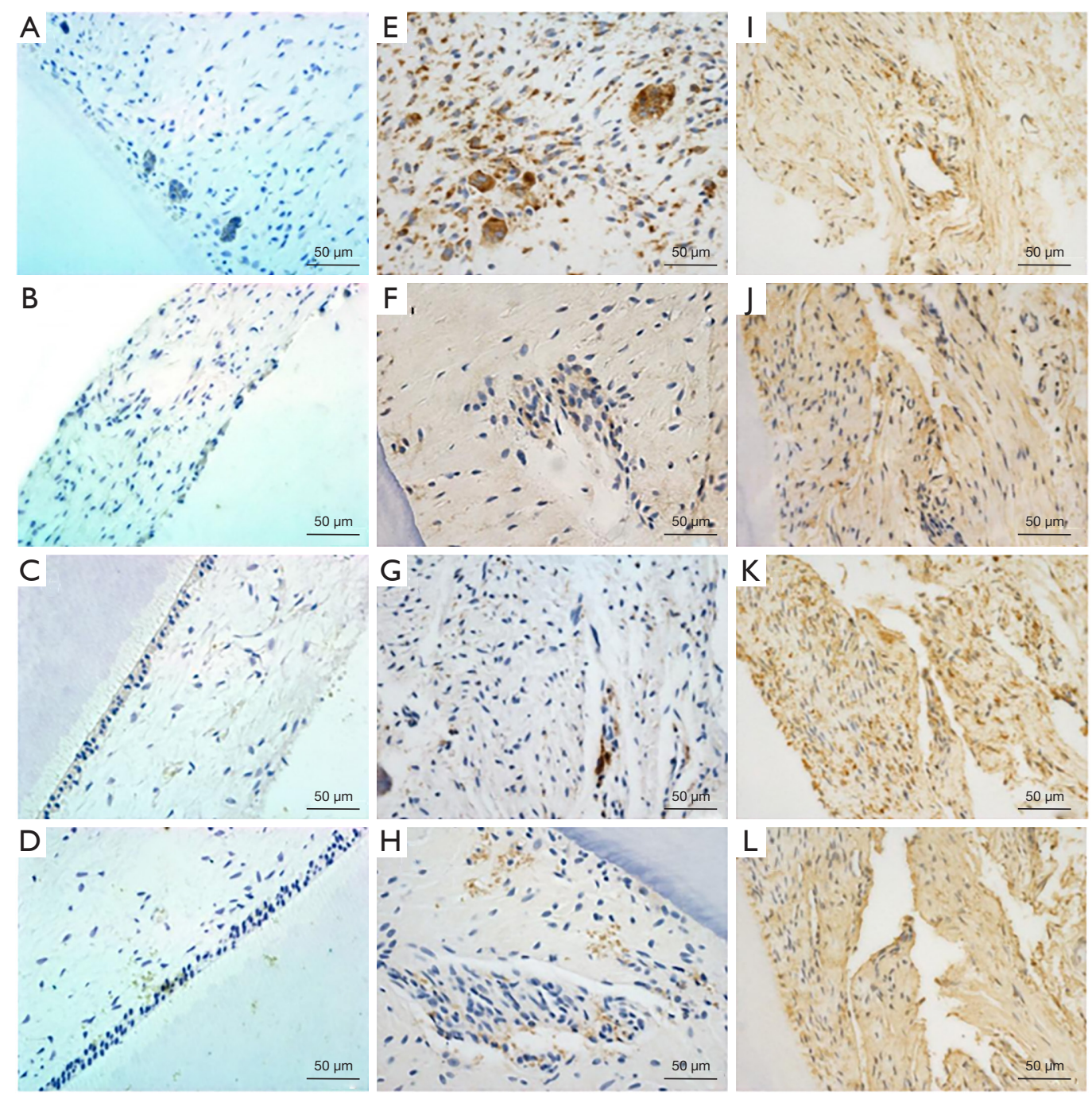

M

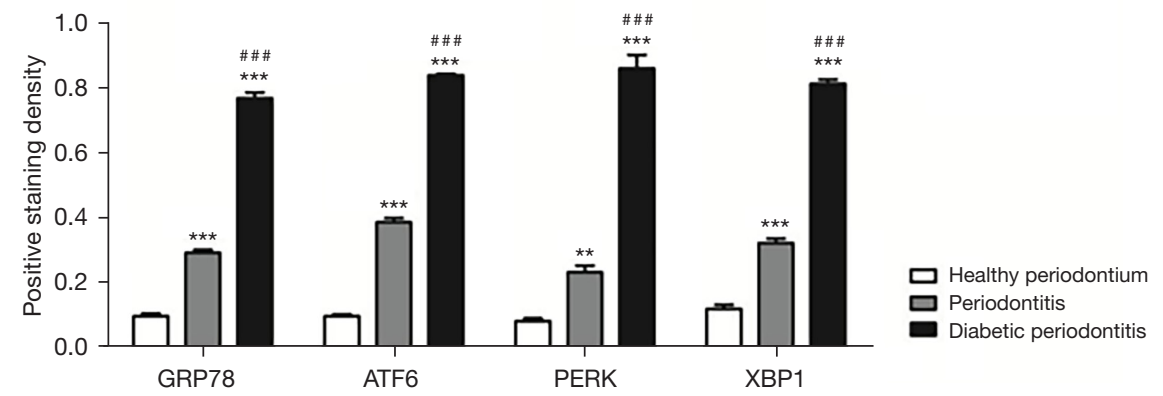

Figure 3 Immunohistochemical staining of ER stress-related factors in periodontal tissues from individuals with healthy teeth, patients with periodontitis, and patients with diabetic periodontitis. GRP78 (A), ATF6 (B), PERK (C), and XBP1 (D) staining of periodontal tissue from healthy group, GRP78 (E), ATF6 (F), PERK (G), and XBP1 (H) staining of periodontal tissue from inflamed group, and GRP78 (I), ATF6 (J), PERK (K), and XBP1 (L) staining of periodontal tissue from diabetic group. (M) Analysis of immunohistochemical staining

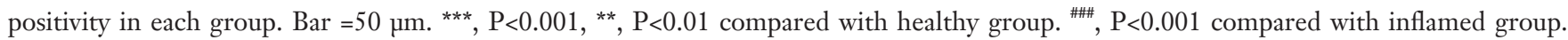
ER, endoplasmic reticulum; GRP78, glucose regulated protein 78; ATF6, activating transcription factor 6; PERK, double-stranded RNAactivated protein kinase (PKR) like endoplasmic reticulum kinase; XBP1, X box-binding protein 1. 

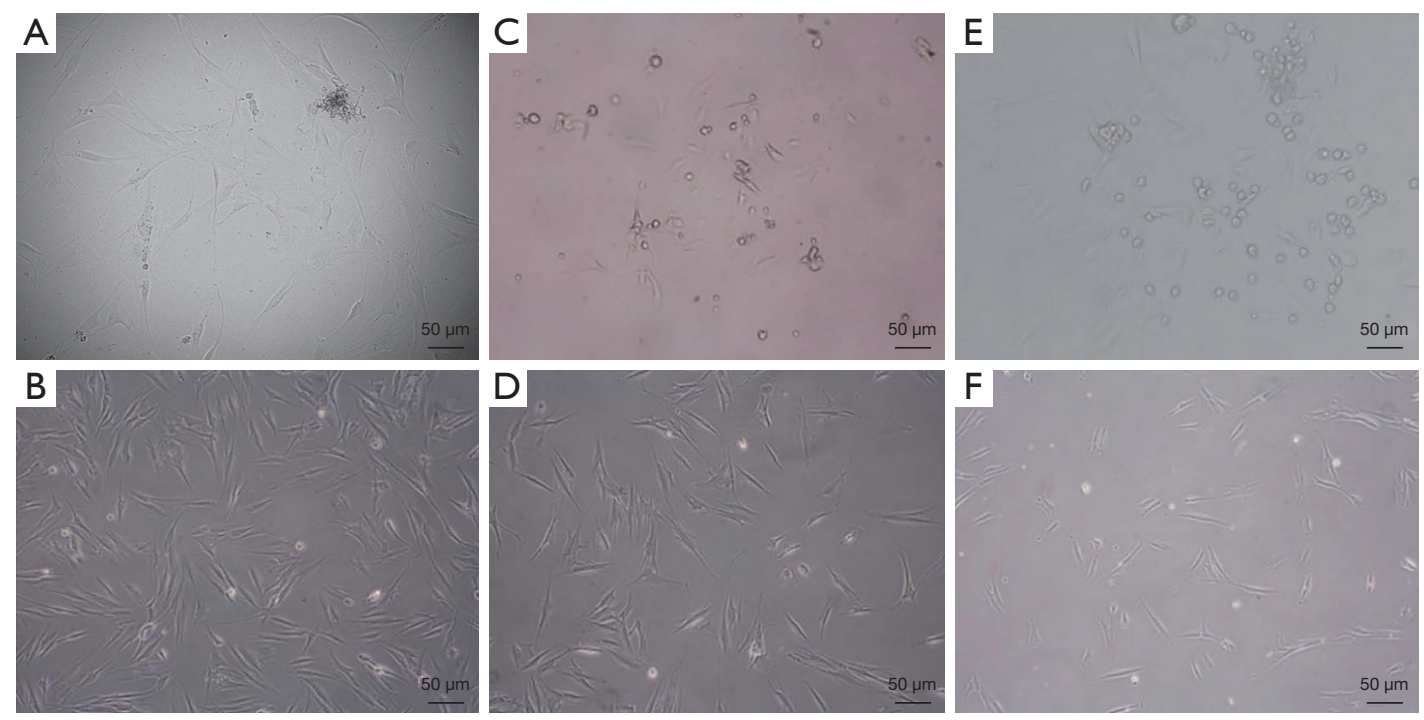

Figure 4 PDLSCs observed with an inverted microscope. (A) Primary PDLSCs from healthy periodontal tissue. (B) Second-generation hPDLSCs after limited dilution and purification. (C) Primary PDLSCs from periodontal tissue of inflamed group. (D) Second-generation iPDLSCs after limited dilution and purification. (E) PDLSCs from periodontal tissue of diabetic group. (F) Second -generation dPDLSCs after limited dilution and purification. Bar $=50 \mu \mathrm{m}$. PDLSCs, periodontal ligament stem cells; hPDLSCs, healthy periodontal ligament stem cells; iPDLSCs, inflamed periodontal ligament stem cells; dPDLSCs, diabetic periodontal ligament stem cells.

\section{With the increase of glucose concentration, the osteogenic differentiation ability of PDLSCs weakens}

PDLSCs from healthy individuals were cultured in medium containing different concentrations of D-glucose $(8,11$, and $25 \mathrm{mmol} / \mathrm{L})$ in vitro to simulate a high glucose microenvironment and allow the changes in the osteogenic differentiation ability of PDLSCs to be observed. PDLSCs treated with $\alpha$-MEM (Minimum Essential Medium Eagle - alpha modification) containing $5.6 \mathrm{mmol} / \mathrm{L} \mathrm{D}$-glucose served as a control group.

After 7 days of culture with D-glucose and osteogenic induction solution, the ALP activities of the cells were observed (Figure 6A-6D). As the D-glucose concentration increased, the ALP activity of osteogenic-induced PDLSCs decreased (Figure $6 E$ ). Cellular proteins were extracted after 14 days of osteogenic induction (Figure $6 F$ ). With the increase of D-glucose concentration, the protein expression levels of Runx2 and OCN in cells in the $25 \mathrm{mmol} / \mathrm{L}$ D-glucose group obviously decreased (Figure 6G). After 4 weeks of osteogenic induction, the results showed that with the increase of D-glucose concentration, the area of positively stained, red mineralized nodules in PDLSCs decreased (Figure 6H-6L).

In summary, with the increase of D-glucose concentration, the osteogenic ability of PDLSCs decreased, and this decrease in osteogenic ability was greatest in PDLSCs stimulated with $25 \mathrm{mmol} / \mathrm{L}$ D-glucose stimulation.

\section{With the increase of glucose concentration, ER stress increases}

PDLSCs from healthy individuals were cultured in D-glucose at different concentrations $(5.6,8,11$, and $25 \mathrm{mmol} / \mathrm{L})$ to simulate a high glucose microenvironment in vitro, and changes in the gene expression of ER stress-related factors were observed at different time points $(6,12,24$, and 72 hours) (Figure 7). As conventional culture medium containing $5.6 \mathrm{mmol} / \mathrm{L}$ of D-glucose, it can be directly used as the $\mathrm{D}$-glucose control group with a concentration of $5.6 \mathrm{mmol} / \mathrm{L}$. QRT-PCR results showed that after stimulation with $8 \mathrm{mmol} / \mathrm{L} \mathrm{D}$-glucose, there were statistical differences in the gene expression of GRP78 at 12 hours, ATF6 at 24 and 72 hours, PERK at 12, 24, and 72 hours, and XBP1 at 24 hours, compared with the control group. However, after stimulation with $11 \mathrm{mmol} / \mathrm{L} \mathrm{D}$-glucose, the gene expression of ER stress-related factors showed statistical difference at each time point compared with the control group, and only XBP1 showed no statistical difference at 6 and 72 hours. After stimulation with $25 \mathrm{mmol} / \mathrm{L} \mathrm{D}$-glucose, the gene expression of ER stress-related factors was statistically 

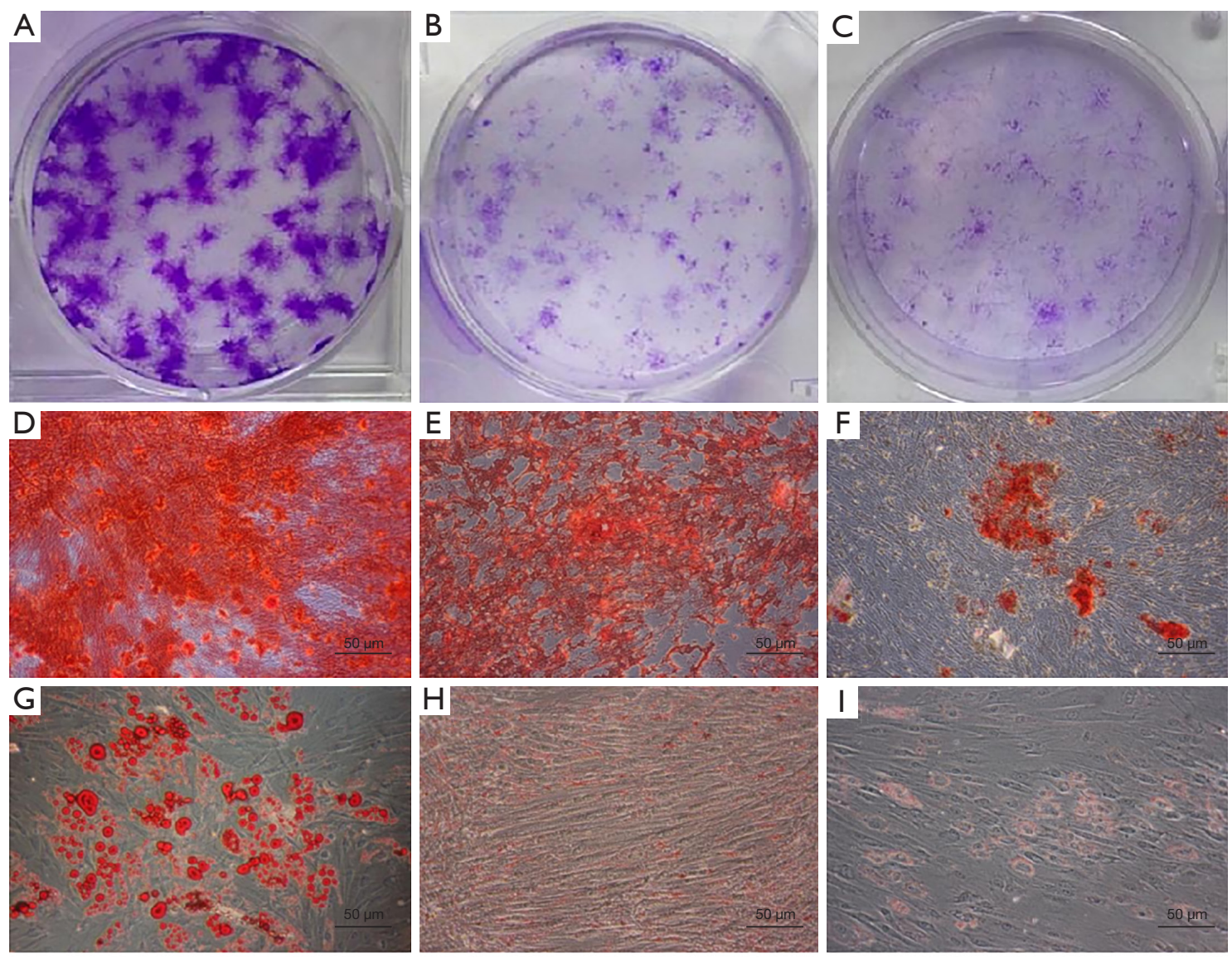

J

K
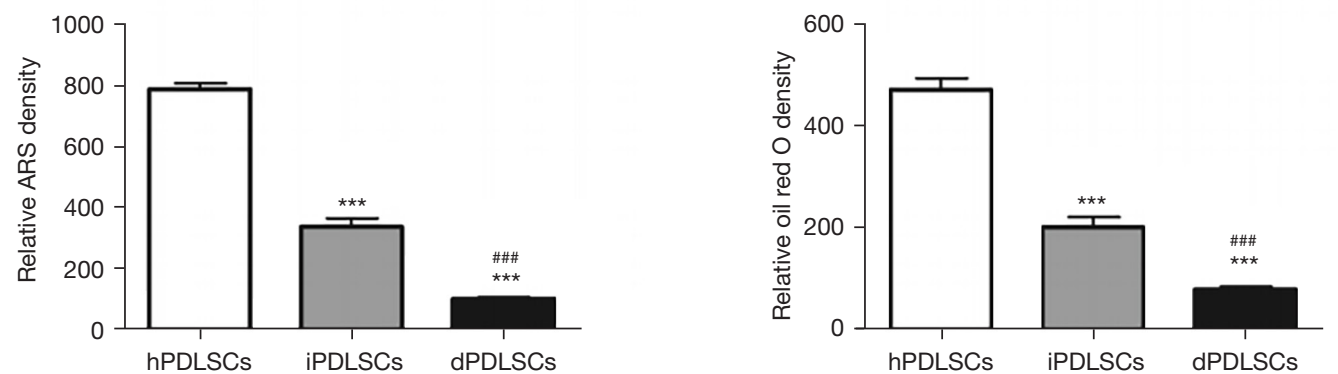

Figure 5 Cell stemness assay of PDLSCs. Clone formation assay of hPDLSCs (A), iPDLSCs (B), and dPDLSCs (C). Alizarin red staining of osteogenic-induced hPDLSCs (D), iPDLSCs (E), and dPDLSCs (F). Bar $=100 \mu \mathrm{m}$. Oil red O staining of adipogenic-induced hPDLSCs $(\mathrm{G})$, iPDLSCs $(\mathrm{H})$, and dPDLSCs (I). Bar $=50 \mu \mathrm{m}$. (J) Comparison of the total optical density values of alizarin red positive staining in each group. (K) Comparison of the total optical density values of Oil red $\mathrm{O}$ positive staining in each group. ${ }^{* * *}$, $\mathrm{P}<0.001$ compared with hPDLSCs. ${ }^{\# \#}, \mathrm{P}<0.001$ compared with iPDLSCs. PDLSCs, periodontal ligament stem cells; hPDLSCs, healthy periodontal ligament stem cells; iPDLSCs, inflamed periodontal ligament stem cells; dPDLSCs, diabetic periodontal ligament stem cells.

different at each time point compared with the control group. When PDLSCs were stimulated with D-glucose at the same concentration for different duration, compared with stimulated for 6 hours, GRP78 gene expression only showed a statistical difference when under stimulated with D-glucose $25 \mathrm{mmol} / \mathrm{L}$ for 12 hours. The gene expression of ATF6 only showed significant differences when $8 \mathrm{mmol} / \mathrm{L} \mathrm{D}$-glucose was used to stimulate cells for 24 and 

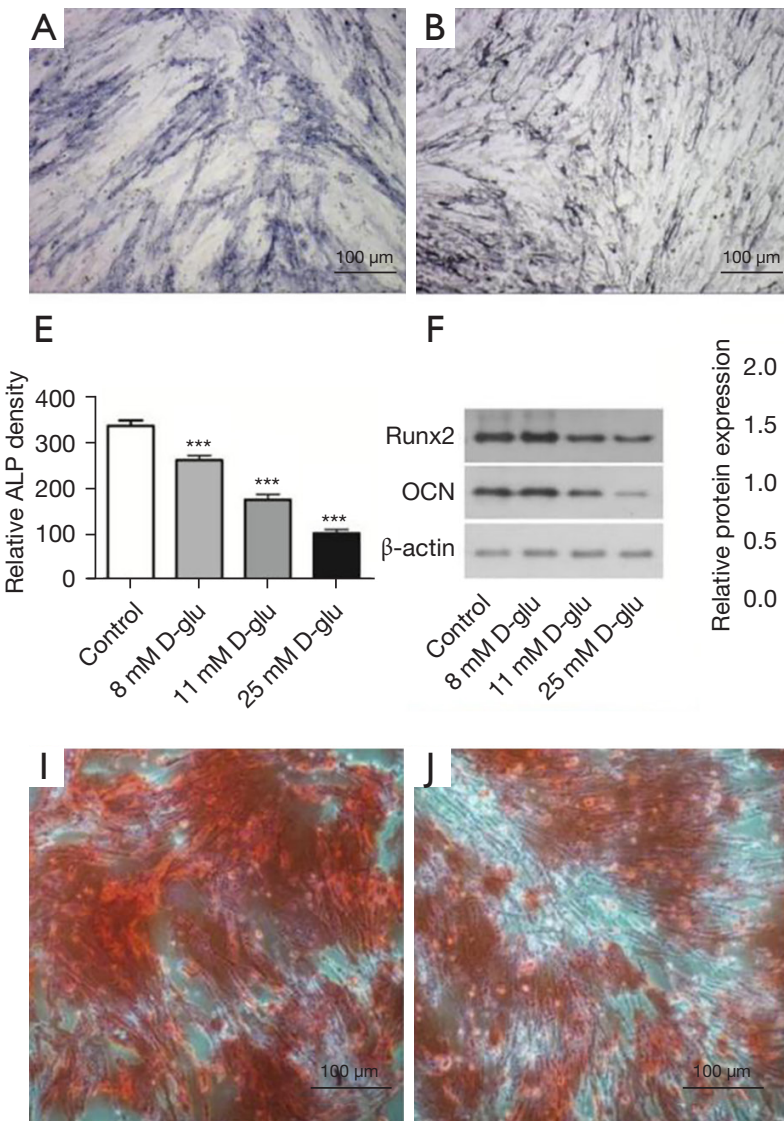

$\mathrm{F}$

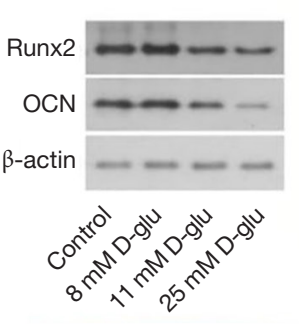

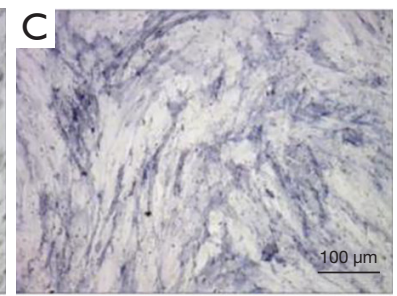

G
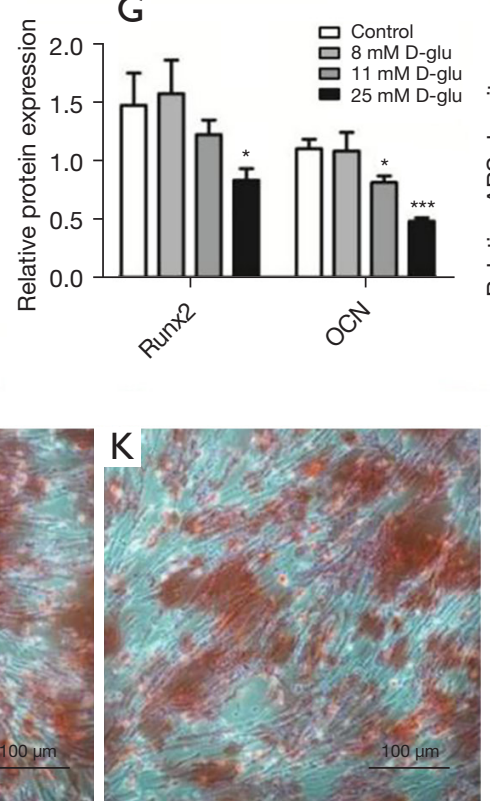

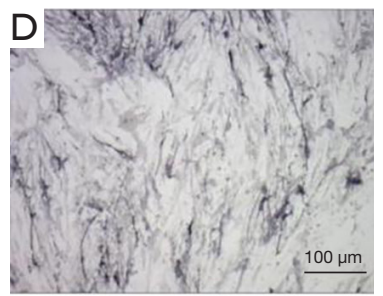

$\mathrm{H}$

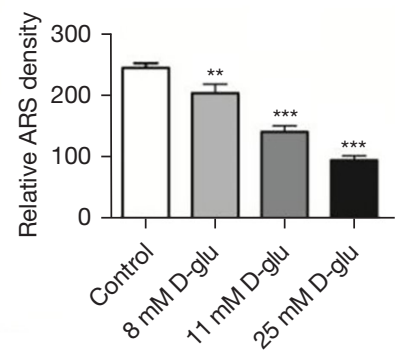

Figure 6 The osteogenic differentiation abilities of PDLSCs cultured in osteogenic inducing medium containing D-glucose at different concentrations. ALP staining of PDLSCs after osteogenic induction in $5.6 \mathrm{mmol} / \mathrm{L}$ D-glucose (control group) (A), 8 mmol/L D-glucose (B), $11 \mathrm{mmol} / \mathrm{L}$ D-glucose (C), and $25 \mathrm{mmol} / \mathrm{L}$ D-glucose (D). (E) Comparison of the total optical density values of ALP positive staining in each group. (F) The protein levels of Runx2 and OCN were detected by western blot after 2-week osteogenic induction at different D-glucose concentrations. (G) Quantification of Runx2 and OCN protein expression. The relative quantities evaluated by band grey value were normalized to $\beta$-actin and are presented as mean $\pm \mathrm{SD}$. (H) Comparison of the total optical density values of alizarin red staining. Alizarin red staining of PDLSCs after osteogenic induction in $5.6 \mathrm{mmol} / \mathrm{L}$ D-glucose (control group) (I), $8 \mathrm{mmol} / \mathrm{L}$ D-glucose (J), $11 \mathrm{mmol} / \mathrm{L}$ D-glucose (K), and $25 \mathrm{mmol} / \mathrm{L}$ D-glucose $(\mathrm{L}) . \mathrm{Bar}=100 \mu \mathrm{m} .{ }^{*}, \mathrm{P}<0.05,{ }^{* *}, \mathrm{P}<0.01,{ }^{* * *}, \mathrm{P}<0.01$ compared with the control group. PDLSCs, periodontal ligament stem cells; ALP, alkaline phosphatase; OCN, osteocalcin; Runx2, runt-related transcription factor 2; SD, standard deviation.

72 hours. The expression of PERK gene showed significant differences under stimulation with 8,11 , and $25 \mathrm{mmol} / \mathrm{L}$ D-glucose at 12 hours, and $25 \mathrm{mmol} / \mathrm{L} \mathrm{D}$-glucose at 24 hours. The gene expression of XBP1 was statistically different only after 24 hours of stimulation with D-glucose at $25 \mathrm{mmol} / \mathrm{L}$. When the $\mathrm{D}$-glucose concentration was $5.6 \mathrm{mmol} / \mathrm{L}$, there was no significant difference in the gene expression of any of the ER stress-related factors between the different timepoints, which showed that ER stress could not be activated by D-glucose at a normal concentration.
To sum up, after 6 hours of stimulation with D-glucose at $25 \mathrm{mmol} / \mathrm{L}$, all ER stress pathways were effectively activated at the gene level. The gene expression levels of the GRP78 and PERK pathways peaked after 12 hours, while those of the IER1 pathway reached their peak at 24 hours. With D-glucose $11 \mathrm{mmol} / \mathrm{L}$ stimulation, the ER stress, and ATF6 and PERK pathways were effectively activated at the gene level after 6 hours, and the gene expression of the IER1 pathway was activated and peaked at 24 hours. With D-glucose stimulation at $8 \mathrm{mmol} / \mathrm{L}$, the ER stress and PERK pathways were effectively activated and reached their 

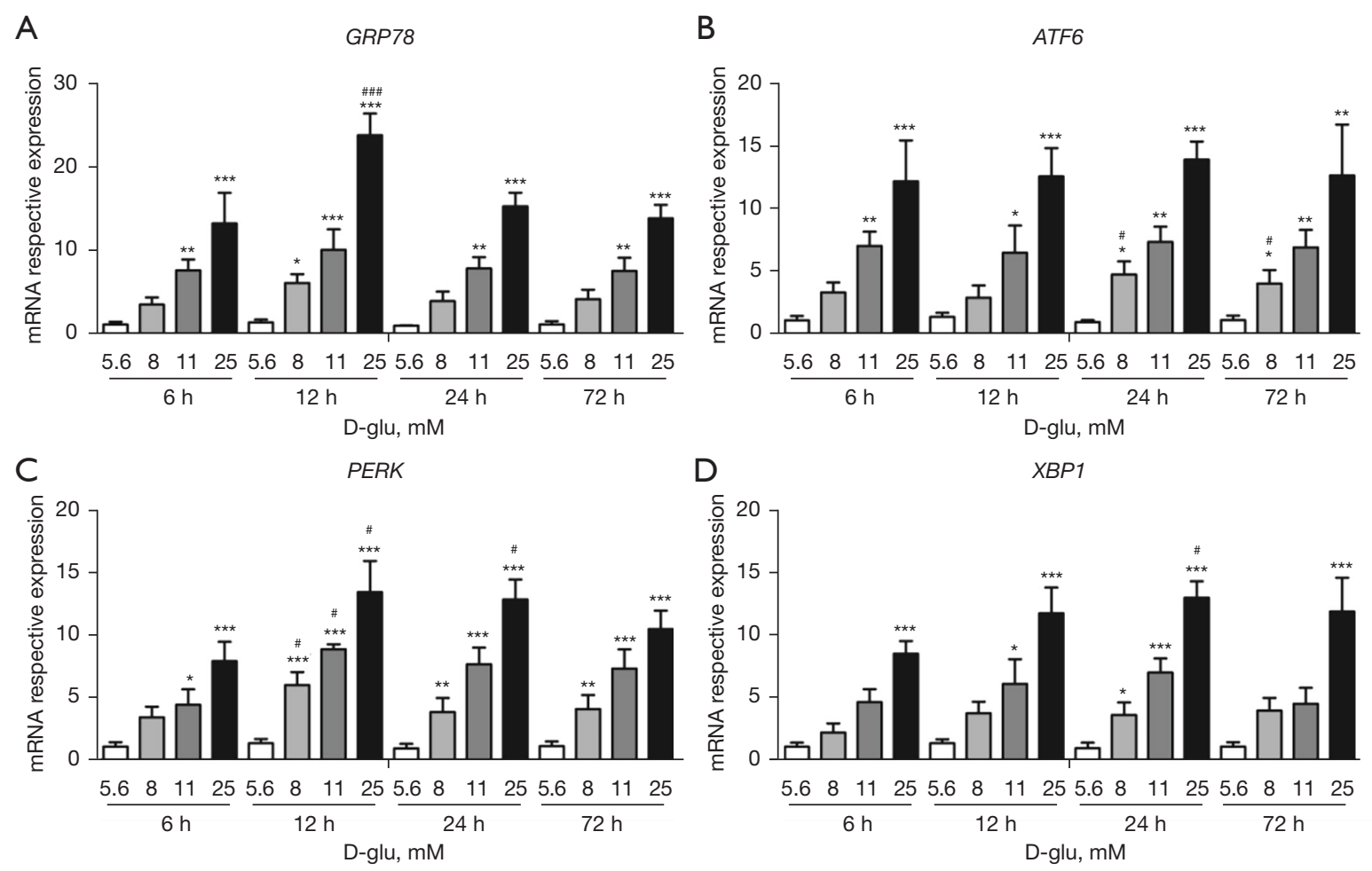

Figure 7 Changes in the expression of ER stress-related genes in PDLSCs after D-glucose stimulation at different concentrations. The mRNA expression of GRP78 (A), ATF6 (B), PERK (C), and XBP1 (D) in PDLSCs after stimulation with different concentrations of D-glucose. * $, \mathrm{P}<0.05,{ }^{* *}, \mathrm{P}<0.01$, ***, $\mathrm{P}<0.001$ compared with the $5.6 \mathrm{mM} \mathrm{D}$-glucose (control) group at the same time point. ${ }^{*}, \mathrm{P}<0.05$, ${ }^{\# \#}$, $\mathrm{P}<0.001$ compared with 6 hours after stimulation with the same D-glucose concentration. ER, endoplasmic reticulum; PDLSCs, periodontal ligament stem cells; mRNA, messenger ribonucleic acid; GRP78, glucose regulated protein 78; ATF6, activating transcription factor 6; PERK, double-stranded RNA-activated protein kinase (PKR) like endoplasmic reticulum kinase; XBP1, X box-binding protein 1.

peak value after 12 hours, while the gene expression of the ATF6 pathway was activated and peaked after 24 hours.

Next, hPDLSCs were cultured with different concentrations of D-glucose (8, 11, and $25 \mathrm{mmol} / \mathrm{L})$ to activate ER stress, and the changes in the protein levels of ER stress-related factors were observed at different timepoints $(6,12,24$, and 72 hours). The concentration of $8 \mathrm{mmol} / \mathrm{L}$ was used as the D-glucose control group. Western blot results (Figure 8) showed that Aa 6 hours, the protein levels of GPR78 and ATF6 under $11 \mathrm{mmol} / \mathrm{L}$ D-glucose stimulation and all the protein levels under $25 \mathrm{mmol} / \mathrm{L}$ D-glucose stimulation were statistically different from those under $8 \mathrm{mmol} / \mathrm{L}$ D-glucose stimulation. At 12 hours, all the protein levels were statistically different only except the level of PERK protein under $11 \mathrm{mmol} / \mathrm{L} \mathrm{D}$-glucose stimulation. At 24 hours, all the protein levels of $25 \mathrm{mmol} / \mathrm{L}$ D-glucose-stimulated cells were statistically different from those of $8 \mathrm{mmol} / \mathrm{L} \mathrm{D}$-glucose groups. At 72 hours, the XBP1 protein levels in the $11 \mathrm{mmol} / \mathrm{L} \mathrm{D}$-glucose stimulation group and all the protein levels under $25 \mathrm{mmol} / \mathrm{L}$ D-glucose stimulation were significantly different from those in the $8 \mathrm{mmol} / \mathrm{L}$ D-glucose stimulation group. After different duration of stimulation with D-glucose at the same concentration, the protein levels of GRP78 and ATF6 were significantly different from those after 6 hours, and PERK protein levels were also significantly different only except stimulated with $11 \mathrm{mmol} / \mathrm{L} \mathrm{D}$-glucose for 72 hours. XBP1 protein levels were only significantly different under stimulation with D-glucose 8,11 and $25 \mathrm{mmol} / \mathrm{L}$ for 12 hours, D-glucose $8 \mathrm{mmol} / \mathrm{L}$ for 72 hours, and D-glucose $25 \mathrm{mmol} / \mathrm{L}$ for 24 hours.

In conclusion, as the glucose concentration increased, ER stress displayed an upward trend. After 6 hours of stimulation with D-glucose $25 \mathrm{mmol} / \mathrm{L}$, the ER stress pathways in PDLSCs were effectively activated at the protein level, and the peak value was reached at 12 hours. 

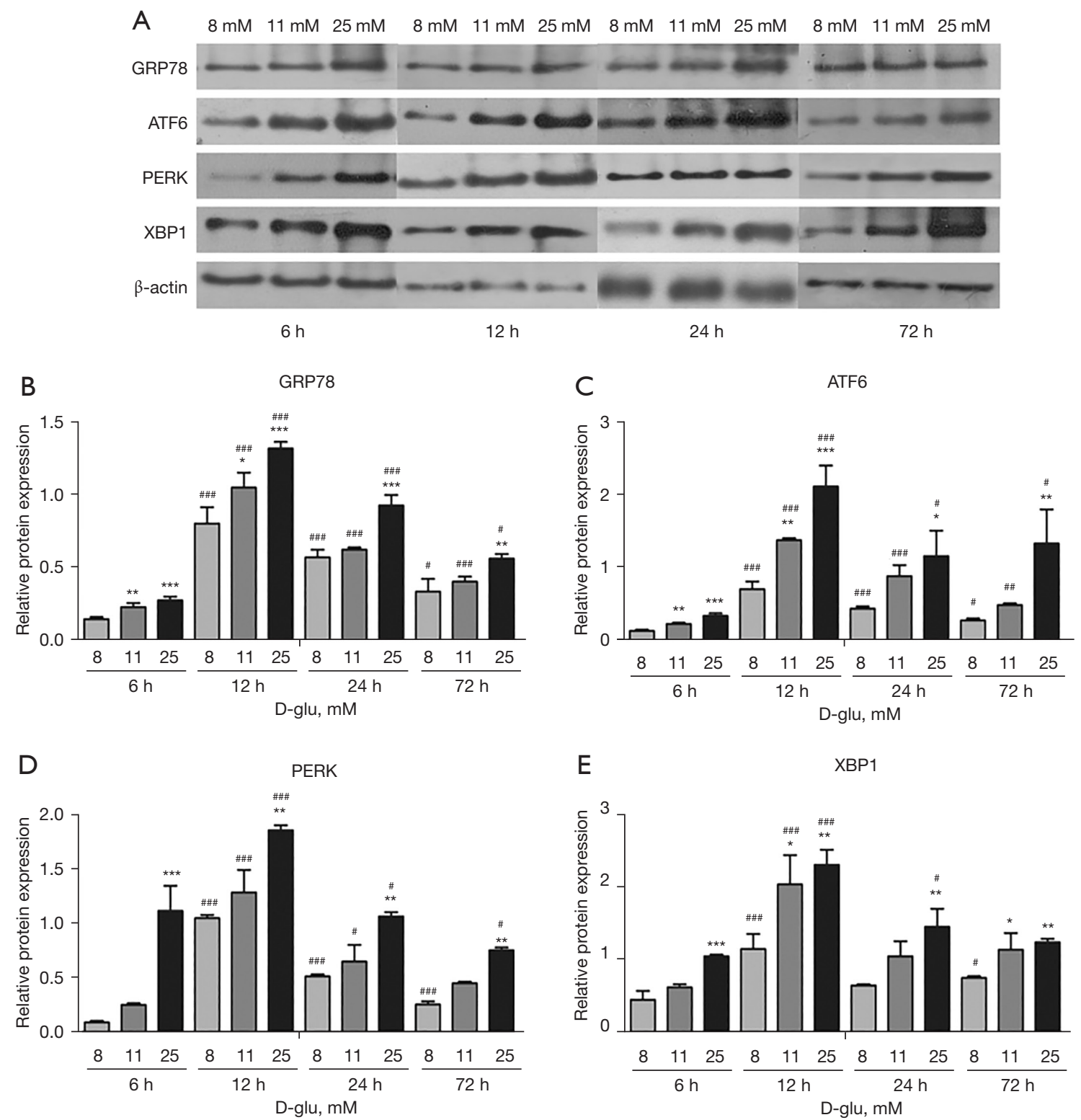

Figure 8 Changes in the protein levels of ER stress-related factors in PDLSCs after stimulation with different concentrations of D-glucose. (A) The protein levels of ER stress-related factors after D-glucose stimulation at different concentrations, as detected by western blot. Quantification of the GRP78 (B), ATF6 (C), PERK (D), and XBP1 (E) protein levels, using $\beta$-actin for internal reference. *, P<0.05, **, $\mathrm{P}<0.01$, ***, $\mathrm{P}<0.001$ compared with $8 \mathrm{mmol} / \mathrm{L} \mathrm{D}$-glucose stimulation group at the same time point. ${ }^{\#}, \mathrm{P}<0.05,{ }^{\#,}, \mathrm{P}<0.01,{ }^{\# \#}, \mathrm{P}<0.001$ compared with 6 hours after stimulation with the same D-glucose concentration. ER, endoplasmic reticulum; PDLSCs, periodontal ligament stem cells; GRP78, glucose regulated protein 78; ATF6, activating transcription factor 6; PERK, double-stranded RNA-activated protein kinase (PKR) like endoplasmic reticulum kinase; XBP1, X box-binding protein 1 .

\section{Blocking ER stress can partially restore the osteogenic differentiation ability of PDLSCs inbibited by a bigh glucose microenvironment}

Based on the results of the presequence experiment, a glucose concentration of $25 \mathrm{nmol} / \mathrm{L}$ was selected for simulation of a high glucose microenvironment in vitro, and the ER stress marker GRP78 shRNA lentivirus was used to block ER stress. The changes in the osteoblast 

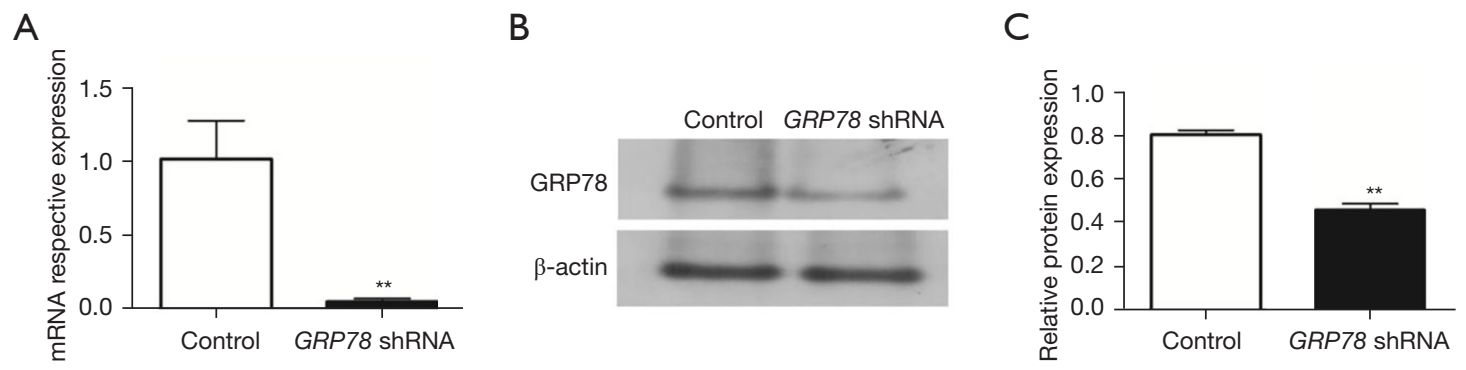

Figure 9 GRP78 expression levels after 48-hour lentivirus infection of PDLSCs cells. (A) The mRNA expressions of GRP78 detected by qRT-PCR. (B) The protein levels of GRP78, as detected by western blot. (C) Quantification of GRP78 protein levels, using $\beta$-actin for internal reference. ${ }^{* *}, \mathrm{P}<0.05$ compared with uninfected controls. GRP78, glucose regulated protein 78; PDLSCs, periodontal ligament stem cells; mRNA, messenger ribonucleic acid; qRT-PCR, quantitative real-time polymerase chain reaction.

differentiation ability of PDLSCs were observed, and the effects of ER stress on periodontal tissue regeneration under high glucose conditions were analyzed.

The mRNA and protein expression levels of GRP78 were detected by qRT-PCR and western blot assay 48 hours after transfection of PDLSCs with GRP78 shRNA lentivirus (Figure 9). The gene expression and protein levels of GRP78 were observed to be significantly decreased compared with those in the control group, and the difference was statistically significant $(\mathrm{P}<0.05)$.

At 48 hours after transfection of PDLSCs with GRP78 shRNA lentivirus, the non-lentiviral transfected control group and the lentiviral transfected group were each divided into two osteogenic induction groups: the $\mathrm{D}$-glucose $8 \mathrm{mmol} / \mathrm{L}$ culture (control) group and the $\mathrm{D}$-glucose $25 \mathrm{mmol} / \mathrm{L}$ culture group. The solution was changed every 3 days.

The gene expression of osteogenic factors was detected by qRT-PCR after 1 week of osteogenic induction (Figure $10 A)$. The mRNA expression levels of Runx2 $(\mathrm{P}<0.001)$ and OCN $(\mathrm{P}<0.01)$ in the $25 \mathrm{mmol} / \mathrm{L} \mathrm{D}$-glucose group were significantly lower than those in the $8 \mathrm{mmol} / \mathrm{L}$ D-glucose control group. After GRP78 shRNA lentivirus transfection, the mRNA expression levels of Runx 2 and $O C N$ significantly increased both in 8 and $25 \mathrm{mmol} / \mathrm{L} \mathrm{D}$-glucose group. There was no significantly different in osteogenic ability-related factors between $25 \mathrm{mmol} / \mathrm{L} \mathrm{D}$-glucose after transfection group and $8 \mathrm{mmol} / \mathrm{L} \mathrm{D}$-glucose control group.

ALP staining results (Figure 10B-10F) showed that PDLSCs in the $25 \mathrm{mmol} / \mathrm{L} \mathrm{D-glucose} \mathrm{group} \mathrm{had}$ significantly reduced ALP activity compared to those in the $8 \mathrm{mmol} / \mathrm{L} \mathrm{D}$-glucose control group $(\mathrm{P}<0.01)$. After GRP78 shRNA lentivirus transfection, ALP activity of cells increased significantly $(\mathrm{P}<0.01)$, and even higher than that in control group $(\mathrm{P}<0.001)$.

Western blot was used to detect the expression levels of Runx2 and OCN protein after 2 weeks of osteogenesis (Figure 10G). In PDLSCs in the $25 \mathrm{mmol} / \mathrm{L} \mathrm{D}$-glucose group, the protein levels of Runx2 and OCN were significantly lower than those in PDLSCs in the $8 \mathrm{mmol} /$ L D-glucose control group $(\mathrm{P}<0.001)$. After GRP78 shRNA lentivirus infection, the protein levels of Runx2 $(\mathrm{P}<0.01)$ and $\mathrm{OCN}(\mathrm{P}<0.05)$ in the $25 \mathrm{mmol} / \mathrm{L} \mathrm{D}$-glucose group significantly increased (Figure 10H), but still lower than those in control group $(\mathrm{P}<0.01,<0.001)$.

After 4 weeks of osteogenic induction, alizarin red staining (Figure 10I-10L) revealed that significantly fewer mineralized nodules had formed in the $25 \mathrm{mmol} / \mathrm{L}$ $\mathrm{D}$-glucose group than in the $8 \mathrm{mmol} / \mathrm{L} \mathrm{D}$-glucose control group $(\mathrm{P}<0.001)$ (Figure 10M). However, after GRP78 shRNA lentivirus transfection, the number of mineralized nodules in the $25 \mathrm{mmol} / \mathrm{L} \mathrm{D}$-glucose group significantly increased $(\mathrm{P}<0.001)$, even had no significantly different with that in control group.

Together, the above results show that blocking the ER stress response with GRP78 shRNA lentivirus resulted in a certain extent of osteogenic capacity restoration of PDLSCs that had been reduced in a high glucose microenvironment (25 mmol/L glucose).

\section{Discussion}

Diabetes is a global epidemic that affects more than 400 million people worldwide (21). It is an endocrine system disease which manifests as abnormally high blood glucose levels (22). Diabetes is associated with many inflammatory response-related complications, including neuropathy, 
A
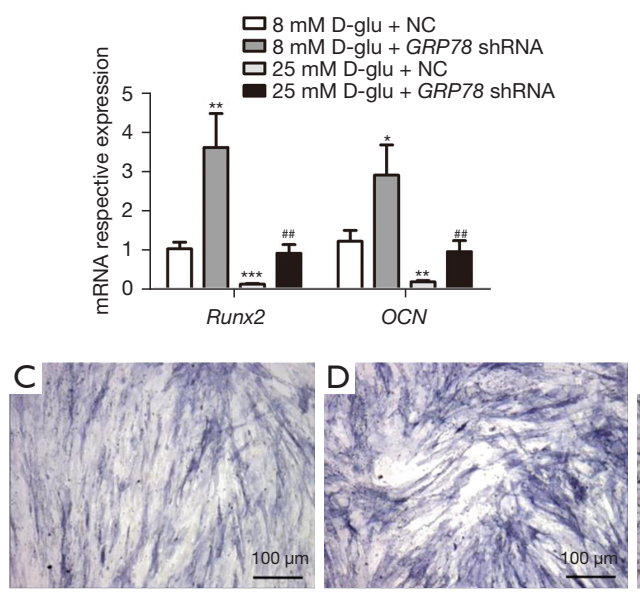

G

D-glu $\quad 8 \mathrm{mM} 8 \mathrm{mM} 25 \mathrm{mM} 25 \mathrm{mM}$ GRP78 ShRNA - $\quad+\quad-\quad+$
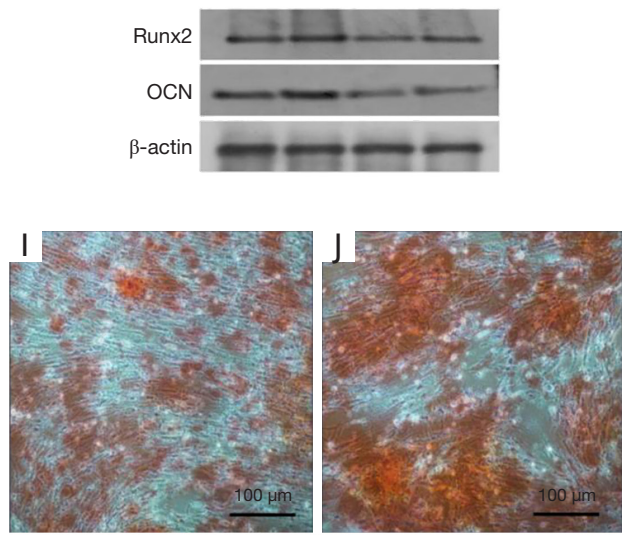

B

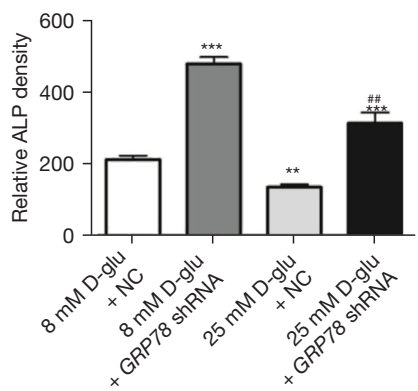

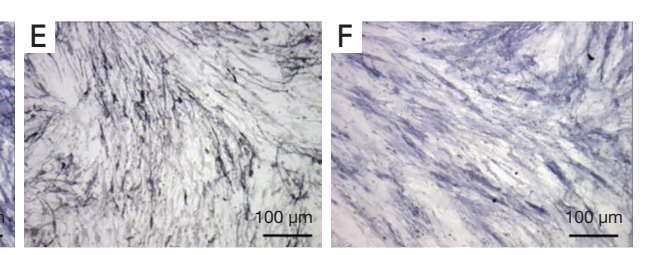

$\mathrm{H}$
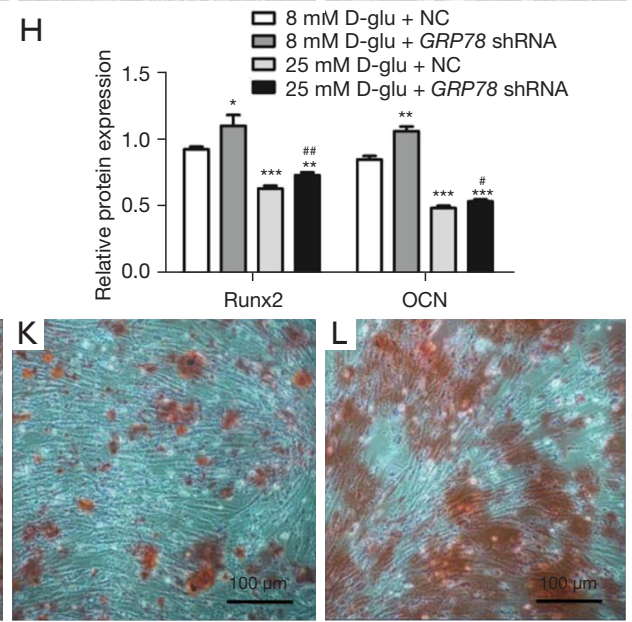

M

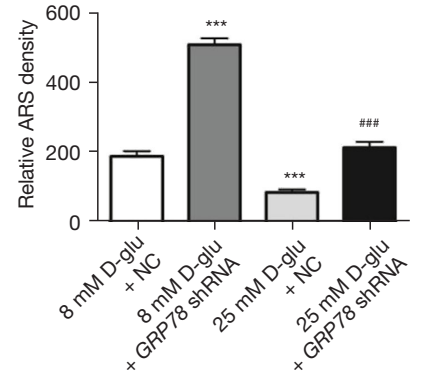

Figure 10 The effects of GRP78 shRNA on the osteogenic differentiation ability of PDLSCs under different glucose concentrations. (A) The mRNA expression levels of osteogenic factors after 7-day osteogenic induction. (B) Comparison of the total optical density values of ALP positive staining in each group. ALP staining of PDLSCs after osteogenic induction in $8 \mathrm{mmol} / \mathrm{L}$ D-glucose + NC (control group) (C), $8 \mathrm{mmol} / \mathrm{L}$ D-glucose + GRP78 shRNA lentivirus (D), $25 \mathrm{mmol} / \mathrm{L}$ D-glucose + NC (E), and $25 \mathrm{mmol} / \mathrm{L}$ D-glucose + GRP78 shRNA lentivirus (F). (G) The protein levels of osteogenic factors, as detected by western blot. (H) Quantification of osteogenic factor protein expression, using $\beta$-actin for internal reference. Alizarin red staining of PDLSCs after osteogenic induction in $8 \mathrm{mmol} / \mathrm{L}$ D-glucose $+\mathrm{NC}$ (control group) (I), $8 \mathrm{mmol} / \mathrm{L} \mathrm{D}$-glucose + GRP78 shRNA lentivirus (J), $25 \mathrm{mmol} / \mathrm{L} \mathrm{D}$-glucose + NC (K), and $25 \mathrm{mmol} / \mathrm{L} \mathrm{D}$-glucose + GRP78 shRNA lentivirus (L). (M) Comparison of the total optical density values of alizarin red positive staining. Bar $=100 \mu \mathrm{m} .{ }^{*}, \mathrm{P}<0.05,{ }^{* *}$, $\mathrm{P}<0.01,{ }^{* * *}, \mathrm{P}<0.001$ compared with $8 \mathrm{mmol} / \mathrm{L} \mathrm{D}$-glucose $+\mathrm{NC}$ (control) group. ${ }^{*}, \mathrm{P}<0.05,{ }^{, \#}, \mathrm{P}<0.01,{ }^{\# \# \#,}, \mathrm{P}<0.001$ compared with $25 \mathrm{mmol} / \mathrm{L}$ D-glucose + NC group. GRP78, glucose regulated protein 78; shRNA, short hairpin ribonucleic acid; PDLSCs, periodontal ligament stem cells; mRNA, messenger ribonucleic acid; ALP, alkaline phosphatase; NC, normal saline. 
nephropathy, retinopathy, cardiovascular disease, and stroke (23-25). Periodontitis is the sixth complication of diabetes, and is more difficult to cure in diabetic patients. Although diabetes and periodontitis are believed to affect each other, the mechanism of interaction between the two is still unclear.

ER stress refers to the accumulation of the unfolded proteins in the ER. In the past decade, ER stress has been proved to play an important role in the occurrence of many inflammatory diseases (26-29). Furthermore, it appears to act as a bridge between diabetes and periodontitis, although the specific relationship between ER stress, diabetes, and periodontitis is not clear.

In this study, we investigated the role of ER stress in diabetes and periodontitis, and discussed the changes in osteogenic differentiation ability and ER stress intensity in PDLSCs under a high glucose microenvironment. Firstly, we obtained periodontal tissues from individuals with healthy teeth, periodontitis patients without systemic diseases, and diabetic patients with periodontitis, and detected the expression levels of ER stress markers. Immunohistochemical and qRT-PCR results showed that the expression of ER stress markers was strongest in diabetic periodontitis followed by simple periodontitis, and was weakest in healthy teeth (Figures 1,3). A previous study has shown that ER stress is enhanced in patients with periodontal disease (30), which is consistent with the results of our study. However, few studies to date have examined the ER stress changes in diabetic periodontitis. The results of this study suggest that ER stress may be involved in the development of diabetic periodontitis.

PDLSCs are MSCs which have multidirectional differentiation ability and play an important role in periodontal tissue regeneration. Existing research shows that diabetes can attenuate the osteogenic differentiation ability of different MSCs through multiple pathways (31). In the current study, we cultured PDLSCs from diabetic patients with periodontitis, periodontitis without systemic disease, and healthy teeth, and tested their cloning, osteogenic, and lipogenic abilities. The results indicate that the cloning, osteogenic, and lipogenic abilities of iPDLSCs and dPDLSCs are decreased, which may be one of the causes of periodontal disease. Research has confirmed that the osteogenic differentiation and adipogenic abilities of iPDLSCs and dPDLSCs are decreased compared with those of healthy PDLSCs (32). In general, the results of our experiment corroborate with those of the earlier study.

In the next part of our study, different concentrations of glucose were used to simulate the blood glucose microenvironment of diabetic patients in order to explore the effect of glucose concentration on the osteogenic differentiation ability of hPDLSCs. The results suggest that high glucose reduces the osteogenic differentiation ability of PDLSCs. The expressions of GRP78, ATF6, PERK, and XBP1 were also detected after exposure to different glucose concentrations for different lengths of time. The results of qRT-PCR and western blot indicated that ER stress can be triggered by a high glucose microenvironment, and that PDLSCs can be activated stably by $25 \mathrm{mmol} / \mathrm{L}$ concentration of D-glucose for 12 hours. We subsequently investigated the changes in the osteogenic differentiation ability of PDLSCs after blocking ER stress with GRP78 shRNA lentivirus at $25 \mathrm{mmol} / \mathrm{L}$ glucose concentration. The results indicate that inhibiting ER stress can promote the recovery of the osteogenic differentiation ability of PDLSCs following exposure to a high glucose microenvironment. Our results are the same as those of an earlier study (33), which showed that the proliferation and osteogenic differentiation abilities of PDLSCs were significantly decreased in $25 \mathrm{mM}$ glucose medium. However, that study explored whether high glucose can inhibit the participation of autophagy in the osteogenic differentiation of PDLSCs, which differs in direction to the current research. Moreover, the results also showed that the osteogenic differentiation ability of PDLSCs only partially recovered when the ER stress was blocked, which suggests that high glucose exerts effects on PDLSCs in various ways. In Chen et al.'s study (34), the mechanism of high glucose $(25 \mathrm{mM})$ inhibiting the differentiation of neural stem cells through oxidative stress and ER stress was explored, and the effect of high glucose on ER stress was also discussed in the experiment. However, the experiment did not set a concentration gradient for high glucose concentration, and focused on other kind of stem cells. Our results showed that high glucose could enhance the ER stress response and inhibit the osteogenic differentiation ability of PDLSCs, and the inhibitory effect increased with the increase of glucose concentration. To date, few studies have directly verified the effects of ER stress in PDLSCs exposed to a high glucose microenvironment. Therefore, our study is innovative in this aspect and has produced clear evidence that the inhibitive effect of high glucose on the proliferation and osteogenic differentiation ability of PDLSCs is partially exerted through stimulation of the ER stress response. 


\section{Conclusions}

In conclusion, this study has uncovered that high blood glucose concentration in diabetic patients reduces the proliferation and osteogenic differentiation ability of PDLSCs by stimulating ER stress, thus exacerbating periodontitis. This finding sheds light on the mechanism of periodontitis associated with diabetes, and may provide a novel viewpoint for the treatment and research of periodontitis in diabetic patients in the future.

\section{Acknowledgments}

Funding: This work was supported by the National Natural Science Foundation of China (No. 81700987).

\section{Footnote}

Reporting Checklist: The authors have completed the MDAR reporting checklist. Available at https://atm.amegroups. com/article/view/10.21037/atm-22-6/rc

Data Sharing Statement: Available at https://atm.amegroups. com/article/view/10.21037/atm-22-6/dss

Conflicts of Interest: All authors have completed the ICMJE uniform disclosure form (available at https://atm. amegroups.com/article/view/10.21037/atm-22-6/coif). The authors report that this work was supported by the National Natural Science Foundation of China (No. 81700987). The authors have no other conflicts of interest to declare.

Ethical Statement: The authors are accountable for all aspects of the work in ensuring that questions related to the accuracy or integrity of any part of the work are appropriately investigated and resolved. All procedures performed in this study involving human participants were in accordance with the Declaration of Helsinki (as revised in 2013). The experimental protocol was approved by the Medical Ethics Committee of the former Guangzhou General Hospital of Guangzhou Military Command (General Hospital of Southern Theater Command of the Chinese People's Liberation Army) (No. 2017-3-6). All study participants gave informed consent to undergo sampling.

Open Access Statement: This is an Open Access article distributed in accordance with the Creative Commons
Attribution-NonCommercial-NoDerivs 4.0 International License (CC BY-NC-ND 4.0), which permits the noncommercial replication and distribution of the article with the strict proviso that no changes or edits are made and the original work is properly cited (including links to both the formal publication through the relevant DOI and the license). See: https://creativecommons.org/licenses/by-nc-nd/4.0/.

\section{References}

1. Slots J. Periodontitis: facts, fallacies and the future. Periodontol 2000 2017;75:7-23.

2. Pihlstrom BL, Michalowicz BS, Johnson NW. Periodontal diseases. Lancet 2005;366:1809-20.

3. Yoshihara A, Seida Y, Hanada N, et al. A longitudinal study of the relationship between periodontal disease and bone mineral density in community-dwelling older adults. J Clin Periodontol 2004;31:680-4.

4. Mulligan R, Phelan JA, Brunelle J, et al. Baseline characteristics of participants in the oral health component of the Women's Interagency HIV Study. Community Dent Oral Epidemiol 2004;32:86-98.

5. Preshaw PM, Bissett SM. Periodontitis and diabetes. $\mathrm{Br}$ Dent J 2019;227:577-84.

6. Chee B, Park B, Bartold PM. Periodontitis and type II diabetes: a two-way relationship. Int J Evid Based Healthc 2013;11:317-29.

7. Stumvoll M, Goldstein BJ, van Haeften TW. Type 2 diabetes: principles of pathogenesis and therapy. Lancet 2005;365:1333-46.

8. Lalla E, Papapanou PN. Diabetes mellitus and periodontitis: a tale of two common interrelated diseases. Nat Rev Endocrinol 2011;7:738-48.

9. Preshaw PM, Alba AL, Herrera D, et al. Periodontitis and diabetes: a two-way relationship. Diabetologia 2012;55:21-31.

10. Nascimento GG, Leite FRM, Vestergaard P, et al. Does diabetes increase the risk of periodontitis? A systematic review and meta-regression analysis of longitudinal prospective studies. Acta Diabetol 2018;55:653-67.

11. Mealey BL, Rose LF. Diabetes mellitus and inflammatory periodontal diseases. Curr Opin Endocrinol Diabetes Obes 2008;15:135-41.

12. Engebretson S, Kocher T. Evidence that periodontal treatment improves diabetes outcomes: a systematic review and meta-analysis. J Clin Periodontol 2013;40 Suppl 14:S153-63.

13. Bascones-Martínez A, González-Febles J, Sanz-Esporrín J. 
Diabetes and periodontal disease. Review of the literature. Am J Dent 2014;27:63-7.

14. Zhang J, Li ZG, Si YM, et al. The difference on the osteogenic differentiation between periodontal ligament stem cells and bone marrow mesenchymal stem cells under inflammatory microenviroments. Differentiation 2014;88:97-105.

15. Li C, Li B, Dong Z, et al. Lipopolysaccharide differentially affects the osteogenic differentiation of periodontal ligament stem cells and bone marrow mesenchymal stem cells through Toll-like receptor 4 mediated nuclear factor $\kappa \mathrm{B}$ pathway. Stem Cell Res Ther 2014;5:67.

16. Yang P, Shen WB, Reece EA, et al. High glucose suppresses embryonic stem cell differentiation into neural lineage cells. Biochem Biophys Res Commun 2016;472:306-12.

17. Oakes SA, Papa FR. The role of endoplasmic reticulum stress in human pathology. Annu Rev Pathol 2015;10:173-94.

18. Cao ZH, Wu Z, Hu C, et al. Endoplasmic reticulum stress and destruction of pancreatic $\beta$ cells in type 1 diabetes. Chin Med J (Engl) 2020;133:68-73.

19. Song X, Li J, Jiao M, et al. Effect of endoplasmic reticulum stress-induced apoptosis in the role of periodontitis on vascular calcification in a rat model. J Mol Histol 2021;52:1097-104.

20. Tan J, Zhou L, Xue P, et al. Tumor Necrosis Factor- $\alpha$ Attenuates the Osteogenic Differentiation Capacity of Periodontal Ligament Stem Cells by Activating PERK Signaling. J Periodontol 2016;87:e159-71.

21. Zimmet P, Alberti KG, Magliano DJ, et al. Diabetes mellitus statistics on prevalence and mortality: facts and fallacies. Nat Rev Endocrinol 2016;12:616-22.

22. Cole JB, Florez JC. Genetics of diabetes mellitus and diabetes complications. Nat Rev Nephrol 2020;16:377-90.

23. Papatheodorou K, Banach M, Bekiari E, et al.

Cite this article as: Tan J, Zhou Y, Luo J, Wu X, Liu H, Wang W, Li Z, Zhong M, Wu L, Li X. High glucose inhibits the osteogenic differentiation of periodontal ligament stem cells in periodontitis by activating endoplasmic reticulum stress. Ann Transl Med 2022;10(4):204. doi: 10.21037/atm-22-6
Complications of Diabetes 2017. J Diabetes Res 2018;2018:3086167.

24. International Diabetes Federation, IDF Diabetes Atlas, International Diabetes Federation, Brussels, Belgium, 7th edition, 2015.

25. Bascones-Martínez A, Muñoz-Corcuera M, BasconesIlundain J. Diabetes and periodontitis: A bidirectional relationship. Med Clin (Barc) 2015;145:31-5.

26. Cao SS, Kaufman RJ. Endoplasmic reticulum stress and oxidative stress in cell fate decision and human disease. Antioxid Redox Signal 2014;21:396-413.

27. Back SH, Kaufman RJ. Endoplasmic reticulum stress and type 2 diabetes. Annu Rev Biochem 2012;81:767-93.

28. Cao SS, Kaufman RJ. Unfolded protein response. Curr Biol 2012;22:R622-6.

29. Wang S, Kaufman RJ. The impact of the unfolded protein response on human disease. J Cell Biol 2012;197:857-67.

30. Domon H, Takahashi N, Honda T, et al. Up-regulation of the endoplasmic reticulum stress-response in periodontal disease. Clin Chim Acta 2009;401:134-40.

31. Tan J, Zhou L, Zhou Y, et al. The Influence of Diabetes Mellitus on Proliferation and Osteoblastic Differentiation of MSCs. Curr Stem Cell Res Ther 2017;12:388-400.

32. Liu Q, Hu CH, Zhou CH, et al. DKK1 rescues osteogenic differentiation of mesenchymal stem cells isolated from periodontal ligaments of patients with diabetes mellitus induced periodontitis. Sci Rep 2015;5:13142.

33. Zhang K, Liu F, Jin D, et al. Autophagy preserves the osteogenic ability of periodontal ligament stem cells under high glucose conditions in rats. Arch Oral Biol 2019;101:172-9.

34. Chen X, Shen WB, Yang P, et al. High Glucose Inhibits Neural Stem Cell Differentiation Through Oxidative Stress and Endoplasmic Reticulum Stress. Stem Cells Dev 2018;27:745-55. 